\title{
OPTIMISING SURVEYS AND RELIABILITY ASSESSMENTS OF HISTORIC CAST-IRON COLUMNS
}

\author{
KAREL JUNG, JANA MARKOVA \& MIROSLAV SYKORA* \\ Klokner Institute, Czech Technical University in Prague, Czech Republic
}

\begin{abstract}
Cast iron structures mostly date back to the second half of the 19th century. Material properties vary due to different composition and production techniques that have been used in different regions over decades. This is why it is of crucial importance to obtain case-specific information on properties of historic structures. Many historic metal structures do not fulfil requirements of present standards. Decisions about adequate construction interventions should be based on complex assessment of structural reliability. In this contribution, the material properties of an iron column are investigated and the key considerations for reliability assessment are presented. Three fundamental issues are addressed to optimise surveys and reliability assessments of cast iron columns: 1 ) an optimum number of material tests; 2) the selection between a finite element (FE) and analytical model; and 3) the target reliability level for a historic structure. It is shown that uncertainties due to limited test data, variability of material and geometrical properties, and resistance model uncertainty can be treated by a semi-probabilistic verification method. As a first approximation, five tensile tests might be considered as an optimum survey strategy. The application of FE models is more demanding than that of simplified analytical formulas, but it can better reflect structure-specific conditions and may increase structural resistance by about $15 \%$. Optimum target reliability level for existing structures with a cultural heritage value could be lower than that for structural design and the assessment value of resistance may increase by about $20 \%$ for a reduced target level.
\end{abstract}

Keywords: heritage structures, reliability assessment, cast iron, structural reliability.

\section{INTRODUCTION}

Most of iron structures date back to the period from the second half of the 19th century to the beginning of the 20th century [1]. Material properties vary due to different composition and production techniques that have been used in different regions. This is why it is of crucial importance to obtain case-specific information on the properties of these historic structures and duly consider the differences between new modern and historic structures in reliability assessments.

This contribution presents an investigation into the material properties of an iron column part of a historic building from 1855. Fig. 1(a)) shows the iron columns under investigation and Fig. 1(b)) displays the damaged column used to prepare specimens for testing. The chemical composition of the alloy was investigated by an optical emission spectrometry excited by glow discharge that conclusively pointed to grey cast iron.

In order to optimise surveys and reliability assessments of cast iron columns, three fundamental questions are addressed in this study:

1. What is an optimum number of destructive tests to estimate tensile and compressive strength of cast iron?

2. What is the benefit when load-bearing capacity of columns is estimated by an advanced Finite Element (FE) model instead of simplified analytical models?

3. Is it reasonable to require the same reliability level for a historic and new structure?

*ORCID: http://orcid.org/0000-0001-9346-3204 


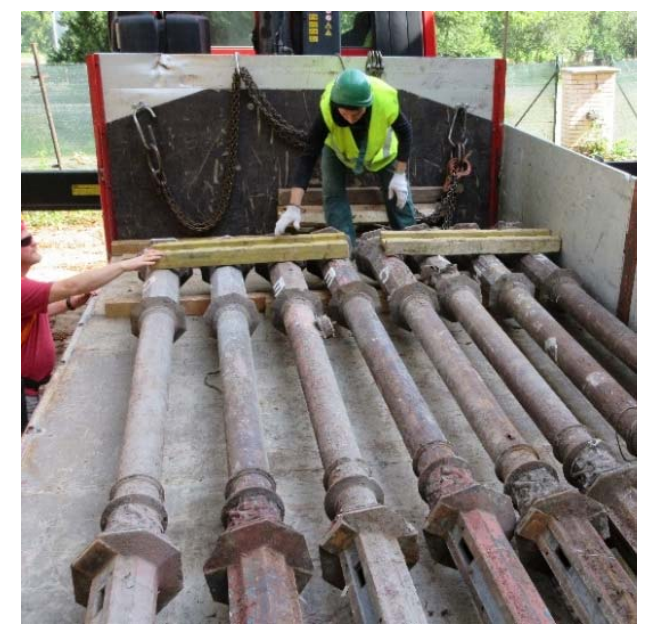

(a)

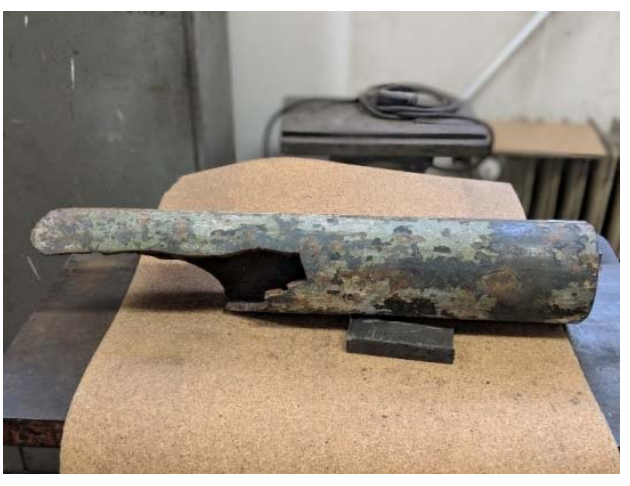

(b)

Figure 1: (a) Iron columns under investigation; (b) Damaged column used for specimens.

The contribution extends the previous study [2] focused on the critical comparison of nondestructive (hardness), minor-destructive (small specimens) and destructive tests of cast iron structures.

\section{OPTIMUM NUMBER OF TESTS}

\subsection{Tensile strength}

Six specimens were prepared for tensile strength tests in accordance with EN ISO 6892-1:2014. One specimen was removed from the sample due to large pores. The five tensile test leads to the following mechanical properties:

- $\quad$ Ultimate tensile strengths $f_{\mathrm{t}, \mathrm{i}}=\{132 ; 169 ; 102 ; 128 ; 101\}$ in MPa with the mean value $\mu_{\mathrm{ft}}=127 \mathrm{MPa}$ and coefficient of variation $V_{\mathrm{ft}}=22 \%$.

- $\quad$ Average ductility $\varepsilon_{\mathrm{t}}=1.7 \%$.

- Mean Young's modulus $\mu_{E}=75 \mathrm{GPa}$ with coefficient of variation $V_{E}=7.9 \%$.

Fig. 2 shows stress and strain diagrams from the tensile tests. The non-homogeneity of the material particularly with respect to $f_{\mathrm{t}}$ and $\varepsilon_{\mathrm{t}}$ is obvious. However, due to a small sample size Grubb's test [3] indicates that the sample likely contains no outlier and extreme observations can result from random variability (confidence level of 0.05 ).

Table 1 provides the overview of iron properties of reported in literature. The investigated material seems to well correspond to cast iron and grey cast iron from Germany and the UK [4]-[7].

The characteristic value of tensile strength can be estimated in accordance with the principles of EN 1990:2002 for basis of design, ISO 13822:2010 and also the Czech standard for assessment of existing structures - CSN 73 0038:2014.

A two-parameter lognormal distribution provides commonly an appropriate model for strengths of steels and historic metal materials [8]. A characteristic value of tensile or compressive strength, $f_{\mathrm{k}}$, is then estimated in accordance with EN 1990: 


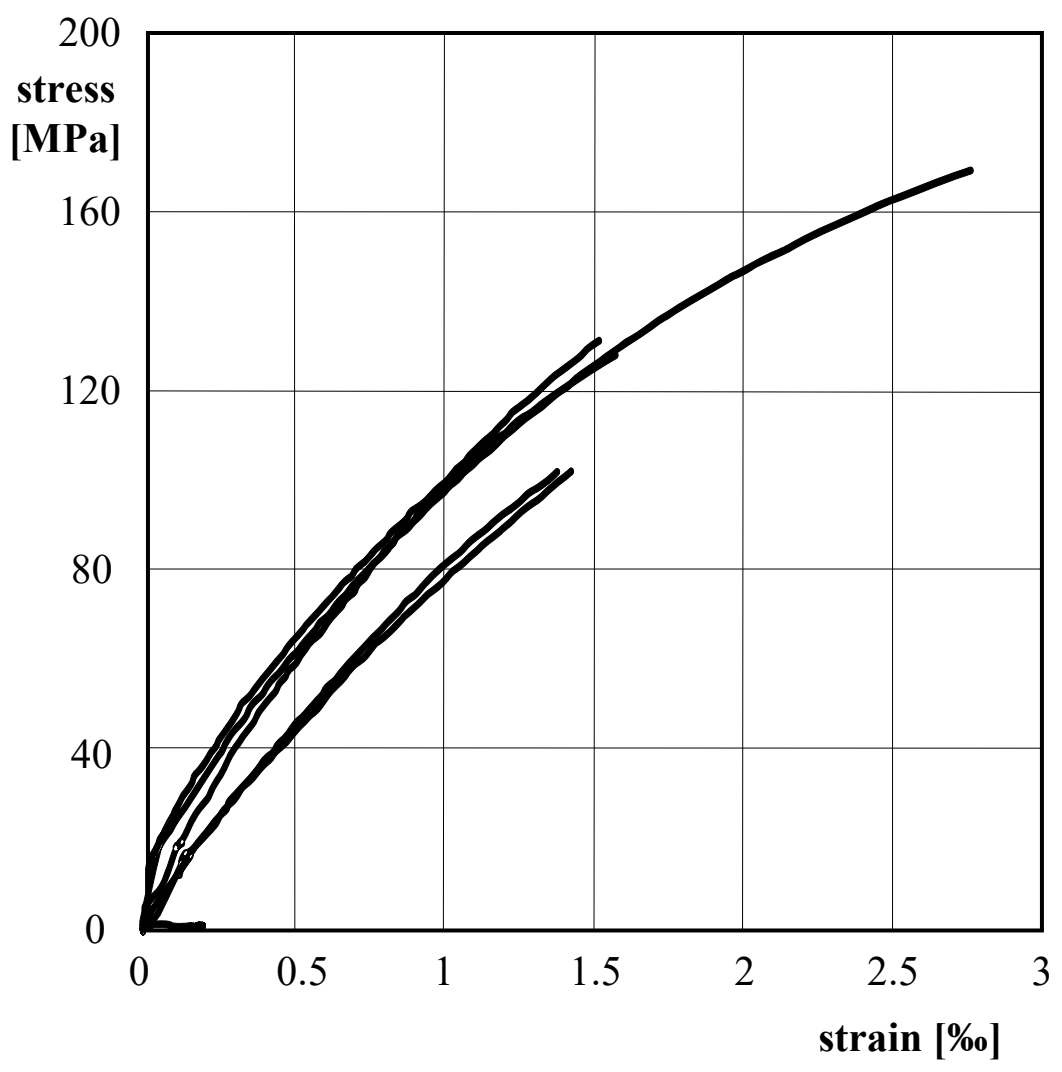

Figure 2: Stress and strain diagrams from the tensile tests.

$$
f_{\mathrm{k}}=\exp \left(m_{\ln f}-k_{n} S_{\ln f}\right) \approx \exp \left(m_{\ln f}-k_{n} V_{f}\right),
$$

where $m_{\ln f}=\sum_{I} \ln \left(f_{i}\right) / n$ (for $i=1 . . n$ and number of tests $n$ ) and standard deviation $s_{\ln f}$ corresponds approximately to the coefficient of variation $V_{f}$. The coefficient $k_{n}$ is accounting for probability associated with estimated fractile (5\% for a characteristic value of a material property) and statistical uncertainty due to a limited number of tests, $n$.

Following the guidance of Annex D of EN 1990 for "unknown $V$ " (when $V_{f}$ is estimated as $\left.s_{f} / m_{f}\right)$, the characteristic value of tensile strength is estimated as follows:

$$
f_{\mathrm{tk}} \approx \exp \left(m_{\ln f \mathrm{t}}-k_{5} V_{\mathrm{ft}}\right)=\exp (4.824-2.33 \times 0.22) \approx 74.5 \mathrm{mPa} .
$$

To provide a first insight into an optimum number of tests for a homogenous material, it is assumed that tests lead to same statistical characteristics of $f_{\mathrm{t}}\left(m_{\ln f \mathrm{t}}\right.$ and $V_{\mathrm{ft}}$ in eqn (2)) but a number of tests is varied from one to ten. Fig. 3 displays the variation of the characteristic tensile strength with $n$ for " $V$ known" and " $V$ unknown". It appears that five to six could be an optimum $n$-value when the coefficient of variation should be estimated from test results. Increasing the number of tests leads to insignificant improvements of the $f_{\mathrm{t}, \mathrm{k}}$-estimate. When the coefficient of variation of strength is assumed to be known (here a conservative value of $25 \%$ is adopted), a number of tests could be reduced to three to four. However, a detailed cost analysis is needed to justify these preliminary observations. 
Table 1: Material properties of iron.

\begin{tabular}{|c|c|c|c|}
\hline Type of iron & Use & Material properties & Ref. \\
\hline Cast & Columns & $\begin{array}{l}f_{\mathrm{t}} \approx 101-169 \mathrm{MPa} \\
f_{\mathrm{c}} \approx 576-785 \mathrm{MPa} \\
E \approx 75 \mathrm{GPa} \\
\varepsilon_{\mathrm{t}} \approx 1.4-2.8 \%\end{array}$ & This study \\
\hline $\begin{array}{l}\text { White of very good } \\
\text { quality, completely fibrous }\end{array}$ & Bridges, truss girders & $\begin{array}{l}f_{\mathrm{t}} \approx 330-360 \mathrm{MPa} \\
\varepsilon_{\mathrm{t}} \approx 6-9 \%\end{array}$ & \multirow{3}{*}{ [1] } \\
\hline $\begin{array}{l}\text { White of ordinary quality, } \\
\text { half-granular, half-fibrous }\end{array}$ & $\begin{array}{l}\text { Girders, angle, } \\
\text { T-profile }\end{array}$ & $\begin{array}{l}f_{\mathrm{t}} \approx 250-320 \mathrm{MPa} ; \\
\varepsilon_{\mathrm{t}} \approx 4-5 \%\end{array}$ & \\
\hline Grey cast of high quality & $\begin{array}{l}\text { Bridges, truss roof } \\
\text { girders }\end{array}$ & $\begin{array}{l}f_{\mathrm{t}} \approx 330-500 \mathrm{MPa} \\
\varepsilon_{\mathrm{t}} \approx 20-31 \%\end{array}$ & \\
\hline Grey cast (Germany) & Columns & $\begin{array}{l}f_{\mathrm{t}} \approx 111-125 \mathrm{Mpa} \\
f_{\mathrm{c}} \approx 448-462 \mathrm{MPa} \\
E \approx 96-111 \mathrm{GPa}\end{array}$ & {$[4]$} \\
\hline Grey cast (UK) & Buildings & $\begin{array}{l}f_{\mathrm{t}} \approx 75-160 \mathrm{MPa} \\
\text { with } \mu_{\mathrm{ft}}=124 \mathrm{MPa} ; \\
f_{\mathrm{c}}=750 \mathrm{MPa} ; \\
E \approx 91 \mathrm{GPa}\end{array}$ & \multirow[t]{2}{*}[5]{,$[6]$} \\
\hline Cast (UK) & Buildings & $\begin{array}{l}f_{\mathrm{t}} \approx 124 \mathrm{Mpa} \\
f_{\mathrm{c}} \approx 590-780 \mathrm{Mpa} \\
E \approx 66-93 \mathrm{GPa}\end{array}$ & \\
\hline Grey cast (Germany) & Columns & $\begin{array}{l}f_{\mathrm{t}} \approx 106-193 \mathrm{MPa} \\
f_{\mathrm{c}} \approx 431-775 \mathrm{MPa} \\
E \approx 91-119 \mathrm{GPa} \\
\varepsilon_{\mathrm{t}} \approx 5 \% \mathrm{o}\end{array}$ & $\begin{array}{l}{[7] \text { and }} \\
\text { references } \\
\text { provided therein }\end{array}$ \\
\hline Cast & NA & $f_{\mathrm{t}} \approx 90-135 \mathrm{MPa}$ & [9] \\
\hline
\end{tabular}

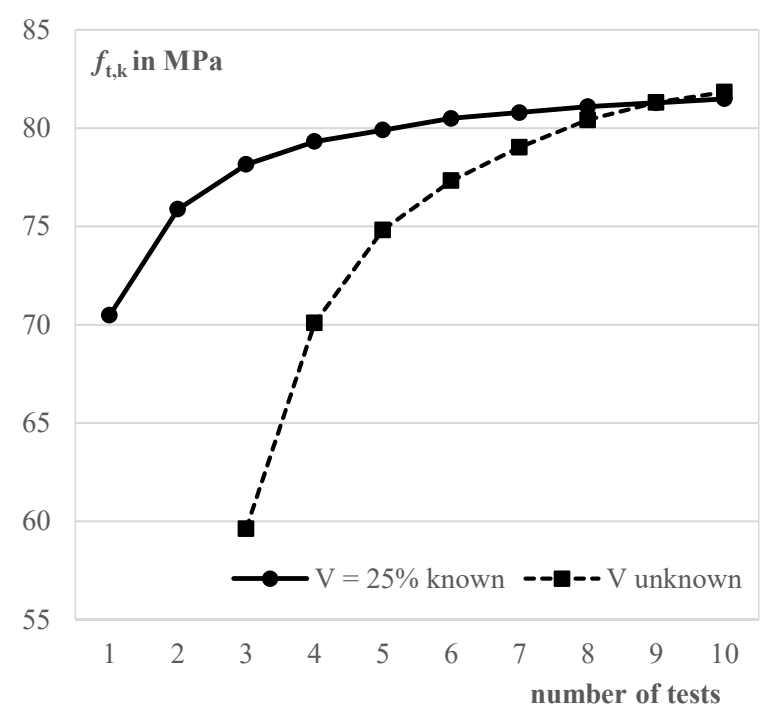

Figure 3: Variation of the characteristic tensile strength with a number of tests. 


\subsection{Compressive strength}

Six small-size specimens - approximate dimensions $10 \times 10 \times 20 \mathrm{~mm}$ - were tested to describe behaviour of iron under compression. The results of compressive strength tests are summarised as follows:

- $f_{\mathrm{c}, i}=\{785 ; 746 ; 633 ; 723 ; 675 ; 576\}$ in MPa.

- Mean value $\mu_{\mathrm{fc}}=690 \mathrm{MPa}$ and coefficient of variation $V_{\mathrm{fc}}=9.9 \%$.

- No outlier detected by Grubb's test.

The ratios $f_{\mathrm{c}} / f_{\mathrm{t}}$ vary from 3.7 to 6.3 (this study 5.4 ) and might be used to estimate tensile strength from compressive strength, the tests of which are based on small-size specimens. Again, the obtained $f_{\mathrm{c}}$-values well correspond to those reported for German and English irons [4]-[7].

Using eqn (1), the characteristic value of compressive strength is obtained as:

$$
f_{\mathrm{ck}} \approx \exp \left(m_{\mathrm{ln} f \mathrm{c}}-k_{6} V_{f_{\mathrm{c}}}\right)=\exp (6.531-2.18 \times 0.099) \approx 553 \mathrm{MPa} .
$$

Assuming again same statistical characteristics of $f_{\mathrm{c}}$ and varying $n$, Fig. 4 shows the variation of the compressive tensile strength with $n$. Depending on the slenderness of a column under investigation, the compressive strength might be less important for structural reliability and even four tests may lead to reasonable accuracy in the case of " $V_{f c}$ unknown". For the case of " $V_{\mathrm{fc}}$ known" ( $15 \%$ conservatively), two or three tests might be sufficient.

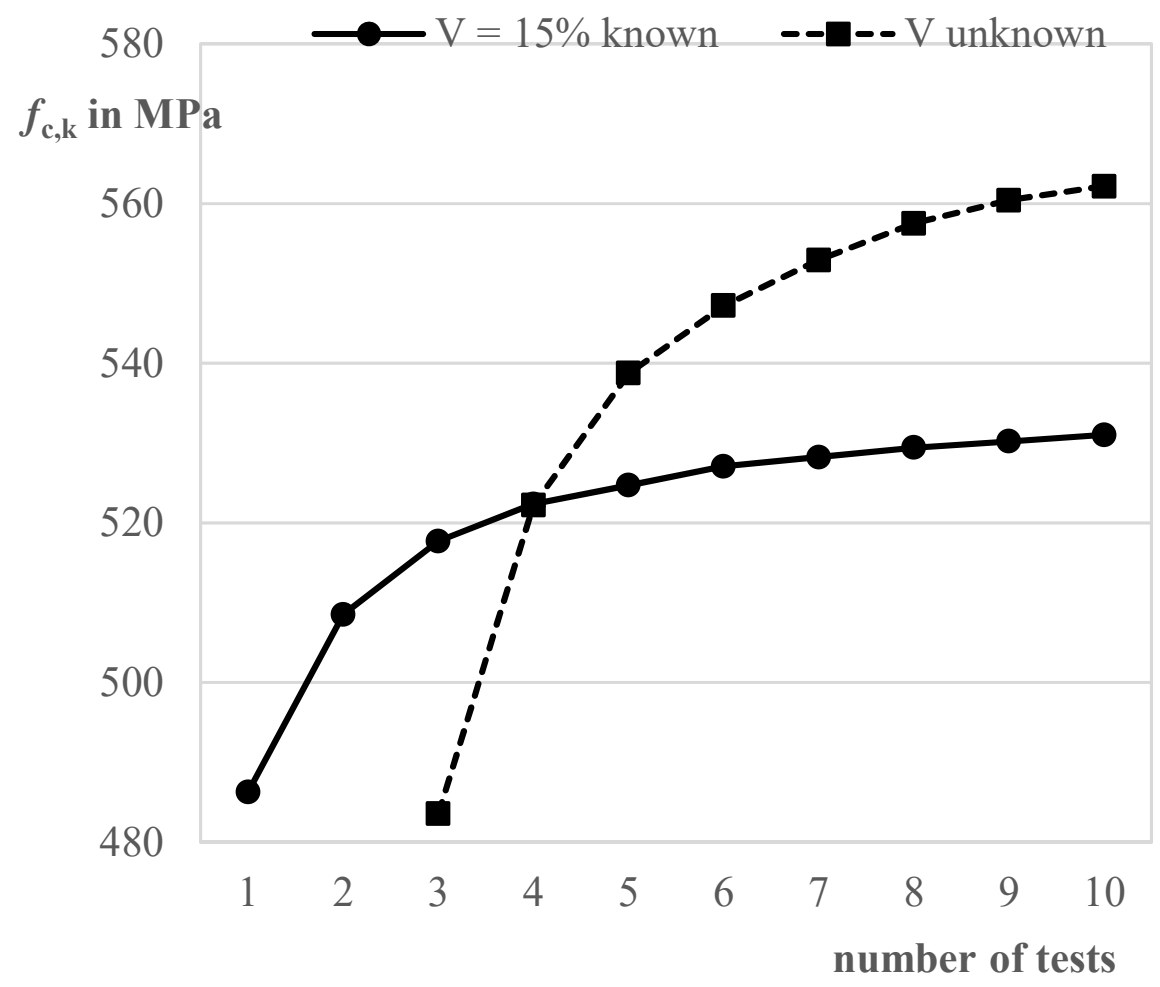

Figure 4: Variation of the characteristic compressive strength with $\mathrm{n}$. 


\section{PARTIAL FACTOR FOR MATERIAL PROPERTY}

Whereas the estimates of the characteristic values may be based on a limited number of tests, the partial factor is commonly based on previous general experience with reliability assessments of steel and historic metal structures and with uncertainties in modelling, material properties and geometry variables, EN 1990 and Caspeele et al. [12]. Following ISO 13822, the Czech standard CSN 730038 provides the following relationship:

$$
\gamma_{\mathrm{M}}=\exp \left(-1.645 V_{f}\right) /\left[\mu_{\xi} \exp \left(-\alpha_{R} \beta V_{R}\right)\right]
$$

where $\alpha_{R}=0.8$ denotes the sensitivity factor for resistance; $\beta=3.8$ is the target reliability index according to EN 1990 and ISO 13822 (see Section 5 for further discussion); $\mu_{\xi}$ the mean of resistance model uncertainty (bias in a structural model, see Holicky et al. [13] for further details); and $V_{R}$ is coefficient of variation of resistance.

In most cases, resistance of the column, $R$, is a linear function of strength $f$, geometrical properties geo (e.g. sectional areas for axial compressive or shear resistances or sectional modulus for flexural resistance), and of uncertainty in the resistance model $\xi$. Coefficient of variation of resistance in eqn (4) can be then estimated as follows:

$$
V_{R} \approx \sqrt{ }\left(V_{f}^{2}+V_{\mathrm{geo}}^{2}+V_{\xi}^{2}\right) .
$$

Table 2 provides an overview of coefficients of variation for historic metallic materials according to CSN 730038 and justification of the values adopted in this study. Using eqn (5), coefficient of variation of resistance becomes:

$$
\begin{gathered}
V_{R \mathrm{t}} \approx \sqrt{ }\left(0.2^{2}+0.05^{2}+0.15^{2}\right)=0.25, \\
V_{R \mathrm{c}} \approx \sqrt{ }\left(0.15^{2}+0.05^{2}+0.15^{2}\right)=0.22 .
\end{gathered}
$$

Partial factors are obtained from eqn (4) disregarding the bias in the structural model $\left(\mu_{\xi}=1\right.$; see the following section):

$$
\begin{gathered}
\gamma_{\mathrm{Mt}}=\exp (-1.645 \times 0.2) / \exp (-0.8 \times 3.8 \times 0.25)=1.54 \\
\gamma_{\mathrm{Mc}}=\exp (-1.645 \times 0.15) / \exp (-0.8 \times 3.8 \times 0.22)=1.53 .
\end{gathered}
$$

The assessment ("design") values are obtained from the characteristic values in eqns (2) and (3):

$$
\begin{gathered}
F_{\mathrm{td}}=f_{\mathrm{tk}} / \gamma_{\mathrm{M}}=74.5 / 1.54=48 \mathrm{Mpa}, \\
F_{\mathrm{cd}}=f_{\mathrm{ck}} / \gamma_{\mathrm{M}}=553 / 1.53=360 \mathrm{Mpa} .
\end{gathered}
$$

As similar values of partial factors are obtained, only the partial factor related to the tensile strength is investigated hereafter only. It is noted that the material factor around 1.5 might be deemed low. In Germany a value of 1.8 might be adopted [4], [7] when small specimens are used for compressive tests and the factor is increased by $10 \%$ implicitly accounting for flaws in the material (caverns, pores, cracks). Further investigations based on more statistical data for material and geometrical properties and load-bearing capacities of columns are needed to refine values of the partial factors for cast iron structures.

The obtained $f_{\mathrm{td}}$ value is in good agreement with CSN 730038 that indicates an assessment value of tensile strength for cast iron $45 \mathrm{MPa}$. The assessment value of $100 \mathrm{MPa}$ indicated in the standard for compressive strength seems to be conservative in comparison to $f_{\text {tc }}$ obtained from tests. Note that CSN 730038 indicates the value of Young's modulus of $100 \mathrm{GPa}$, which is slightly higher than that obtained in this study. 
Table 2: Coefficients of variation for structural members from historic metals.

\begin{tabular}{|c|c|c|l|}
\hline Symbol & $\begin{array}{c}\text { Coefficient of } \\
\text { variation according } \\
\text { to CSN 73 0038 }\end{array}$ & $\begin{array}{c}\text { Adopted } \\
\text { value }\end{array}$ & Justification \\
\hline$V_{f t}$ & $0.10-0.15$ & 0.2 & $\begin{array}{l}\text { The value estimated from tensile tests, 0.22, } \\
\text { seems to be slightly conservative for } \\
\text { common cast irons. While a conservative } \\
\text { estimate of } V_{f} \text { is normally adopted to } \\
\text { estimate } f_{\mathrm{k}} \text { for a few tests (Section 2), a } \\
\text { reasonable value should be selected to } \\
\text { derive a representative partial factor. }\end{array}$ \\
\hline$V_{f \mathrm{c}}$ & $0.10-0.15$ & 0.15 & $\begin{array}{l}\text { The lower bound of the range indicated in } \\
\text { CSN 73 0038 well corresponds to the } \\
\text { estimate from tests. } V_{f \mathrm{c}}=0.15 \text { is deemed to } \\
\text { be reasonable as the compressive strength } \\
\text { of iron exhibits commonly lower dispersion } \\
\text { in comparison to the tensile strength. }\end{array}$ \\
\hline$V_{g e o}$ & $0.05-0.10$ & 0.05 & $\begin{array}{l}\text { Dimensions are verified in-situ; the lower } \\
\text { bound is thus considered. See [7] for further } \\
\text { information on the variability of dimensions } \\
\text { of cast iron structural members. }\end{array}$ \\
\hline$V_{\xi}$ & $0.05-0.10$ & 0.15 & $\begin{array}{l}\text { The estimate in CSN 73 0038 assumes an } \\
\text { unbiased mechanical model }\left(\mu_{\xi}=1 \text { in }\right. \\
\text { eqn (4)) Here } V_{\xi}=0.15 \text { obtained in [10], } \\
\text { [11] for the analytical model of resistance } \\
\text { of slender iron columns [5], [6] is } \\
\text { considered. }\end{array}$ \\
\hline
\end{tabular}

4 CHOICE BETWEEN ANALYTICAL AND NUMERICAL MODEL

In analogy with the estimates of load bearing capacity of steel columns, analytical models are available for centrically loaded cast iron columns:

- Heyde and Lindner [4], [7] adapted a reduction factor $\chi$, used for steel members, to verify buckling of geometrically imperfect iron columns on the basis of their sectional area and compressive strength.

- Rondal and Rasmussen [5], [6] developed a more complex model that accounts for the geometrical imperfections of cast-iron columns, the tensile strength significantly lower than the compressive strength, non-linear stress-strain curve and a low Young's modulus by checking failure due to yielding in compression and fracture in tension.

While the uncertainty associated with Heyde and Lindner's model has not been investigated in detail yet, Brych et al. [10] and Brych and Sykora [11] compared the predictions of Rondal and Rasmussen's model with the results of 72 full-scale tests of columns made of English iron and English grey cast iron. They derived $\mu_{\xi}=1.2-1.25$ depending on slenderness and $V_{\xi}=0.15$. The model thus seems to systematically underestimate load bearing capacity (by about 20-25\%). However, this "hidden safety" is normally disregarded in reliability verifications and the reduction by $\mu_{\xi}$ is not commonly utilised in eqn (4). Moreover, the validity of the bias for irons produced outside of the UK should be verified focusing on the effects of the porosity of a material, geometrical imperfections, and $f_{\mathrm{c}} / f_{\mathrm{t}}$ ratio. 
For illustrative purposes, it is assumed that Rondal and Rasmussen's model underestimates a true characteristic resistance of columns, $R_{\mathrm{k}}\left(f_{\mathrm{tk}} ; f_{\mathrm{ck}}\right)$, by $20 \%$ and the assessment value of resistance is derived using the partial factor $\gamma_{\mathrm{M}} \approx 1.55$ for $V_{\xi}=0.15$; see eqns (8) and (9):

$$
R_{\mathrm{d}}\left(f_{\mathrm{tk}} ; f_{\mathrm{ck}}\right) \approx 0.8 R_{\mathrm{k}}\left(f_{\mathrm{tk}} ; f_{\mathrm{ck}}\right) / 1.55=R_{\mathrm{k}}\left(f_{\mathrm{tk}} ; f_{\mathrm{ck}}\right) / 1.94 .
$$

Heyde and Lindner [4], [7] predicted resistances of cast iron columns using a FE model to reflect the geometrical and material non-linearity. They reached a very good match between test and model results; the comparison of 12 test and FE model results yields $\mu_{\xi}=1.1$ and $V_{\xi}=0.12$ which are typical values for the resistance models of steel members [14], [15].

In comparison to the analytical models, the application of the FE model is more timedemanding, but the model is capable to better reflect the complex geometry of columns, different material properties (e.g. shape of stress-strain diagram), local geometrical imperfections and defects, and larger eccentricity of loading. Considering $V_{\xi}=0.12$, eqns (6) and (8) lead to $V_{R \mathrm{t}} \approx V_{R}=0.24$ and $\gamma_{\mathrm{Mt}} \approx \gamma_{\mathrm{M}}=1.49 \approx 1.5$. The assessment value derived using the FE model is about $15 \%$ larger in comparison to that obtained by the analytical model:

$$
R_{\mathrm{d}}\left(f_{\mathrm{tk}} ; f_{\mathrm{ck}}\right) \approx 0.9 R_{\mathrm{k}}\left(f_{\mathrm{tk}} ; f_{\mathrm{ck}}\right) / 1.5=R_{\mathrm{k}}\left(f_{\mathrm{tk}} ; f_{\mathrm{ck}}\right) / 1.67 \text {. }
$$

This is why it is recommended to start by verifying reliability of columns by a simplified analytical model and then, if needed, proceed with the application of a FE model, keeping in mind the expected difference should be around $15 \%$ and larger differences should be explained.

\section{TARGET RELIABILITY LEVEL}

\subsection{New versus existing structures}

The CEN Technical Specification [16] recommends that target reliability levels, $\beta$, should reflect the type and importance of the structure, possible failure consequences and socioeconomic criteria. $\beta$-levels for existing structures can be different from those for structural design due to the following considerations:

- Economic: the relative cost of safety measures in existing structures can be very high.

- Societal: possible resettlement of inhabitants, relocation of activities or loss of public productivity, economic impact and loss of heritage values must be considered in relation to existing structures.

- Sustainability considerations: repair or upgrading of existing structures normally enables the choice of the most appropriate materials and implies reduction in the use of resources compared to replacement by new structures.

fib bulletin 80 for assessment of existing concrete structures [17] makes distinction between $\beta$-levels for:

1. Reliability assessment of an existing structure.

2. Optimum upgrade of the structure.

While the levels for an optimum upgrade are in general close to those accepted for structural design, $\beta$-values for the assessment may be substantially lower [17]-[19]. As an example the value of 3.8 could be reduced to 2.3 for moderate consequences of failure (CC2 in EN 1990). For high failure consequences $\mathrm{CC} 3$, a 50 -year value of 4.3 given in the Eurocode might be reduced to 2.8 according to the bulletin. 


\subsection{Consideration of the cultural heritage value}

According to the CEN Technical Specification [16], the target reliability may be specified on a project specific basis taking into account cultural and societal aspects. The fundamental principles for the assessment of heritage structures are provided in Annex I of ISO 13822. Construction works and also their load-bearing structures often represent an important aspect of the culture of a certain time period. Structures can support other character-defining elements such as technologies, facades or other non-structural members including historic mural paintings.

An attempt to indicatively classify consequence classes, and thus thereby target levels, with respect to heritage values was made in [8], [20] - see Table 3. As an example the table recognises that a residential building normally classified in CC2 should be considered as a CC3 structure when it is of a high cultural heritage value. The technical possibilities of preserving the heritage value are taken into account as a secondary aspect - the structure is in $\mathrm{CC} 3$ for normal and high possibilities while it could be downgraded to CC2 when it is technically difficult to maintain a reliability level associated with CC3. See [21] for further details.

\subsection{Variation of partial factor with target reliability}

With reference to eqns (4), (12) and (13), Fig. 5 shows the effect of target reliability on the ratio $R_{\mathrm{d}} / R_{\mathrm{k}}$. It appears that it is of uttermost importance to specify target reliabilities adequate for existing structures with a cultural heritage value. Considering the results for a FE model, the change in $\beta$ from 3.8 to 2.8 implies the increase of the assessment value by about $20 \%$ $\left(R_{\mathrm{d}} / R_{\mathrm{k}}\right.$ increasing from 0.6 to 0.73$)$.

\section{CONCLUSIONS}

Numerous uncertainties affect resistances of cast iron columns. Uncertainties due to limited test data, variability of material and geometrical properties, and resistance model uncertainty can be treated by a semi-probabilistic verification method that is suitable for practical applications. Three fundamental issues need to be addressed to optimise surveys and reliability assessments of cast iron columns:

1. It appears that five to six could be an optimum number of material tests. When the coefficient of variation of iron strength is known, a number of tests could be reduced to about three. A detailed cost analysis is needed to justify these preliminary observations, however.

Table 3: Indicative relation between the heritage value of a building or bridge and the Consequence Class according to EN 1990 - minimum requirement on CC.

\begin{tabular}{|l|c|c|c|}
\hline \multirow{2}{*}{ Technical possibilities of preserving heritage value } & \multicolumn{3}{|c|}{ Heritage value } \\
\cline { 2 - 4 } & Low & Medium & High \\
\hline Small & CC1 & CC1 & CC2 \\
\hline Medium & CC1 & CC2 & CC3 \\
\hline High & CC2 & CC3 & CC3 \\
\hline
\end{tabular}




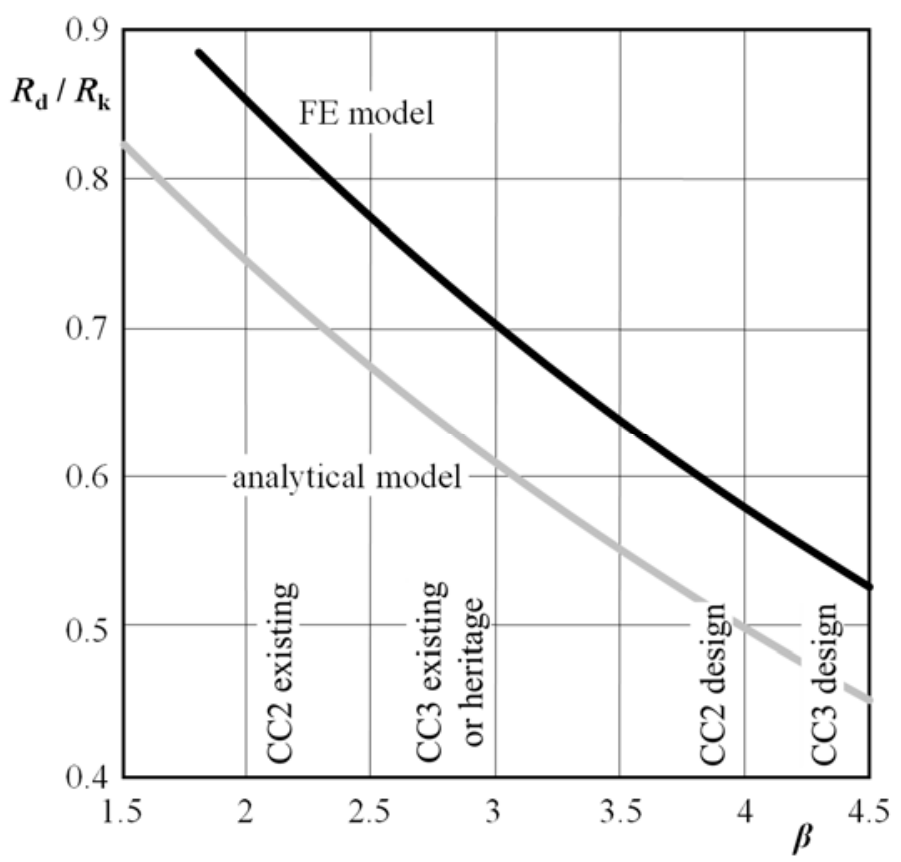

Figure 5: Variation of the assessment-to-characteristic resistance with the target reliability.

2. The application of FE models is more demanding than that of simplified analytical formulas, but it can better reflect structure-specific conditions and may increase structural resistance by about $15 \%$. This is why it is recommended to start by verifying reliability using an analytical model and then, if needed, proceed with the application of a FE model.

3. When the optimised target reliability level for existing structures with a cultural heritage value is considered instead of that for structural design, structural resistance increases by about $20 \%$. Therefore, it is important to specify in standards and apply in reliability assessments target reliabilities adequate for existing structures, possibly with a cultural heritage value.

\section{ACKNOWLEDGEMENT}

This study is a part of the project NAKI DG16P02M050 "Optimisation of observations and assessment of heritage structures", supported by the Ministry of Culture of the Czech Republic.

\section{REFERENCES}

[1] de Bouw, M., Wouters, I., Vereecken, J. \& Lauriks, L., Iron and steel varieties in building industry between 1860 and 1914: A complex and confusing situation resolved. Construction and Building Materials, 23(8), pp. 2775-2787, 2009. DOI: 10.1016/j.conbuildmat.2009.03.009.

[2] Jung, K., Markova, J. \& Sykora, M., Evaluating strength of historic cast iron using destructive and non-destructive tests (under review). Proceedings of the 16th International Probabilistic Workshop, pp. 161-172, 2018. 
[3] Holicky, M., Introduction to Probability and Statistics for Engineers, Springer-Verlag: Berlin, 181 pp., 2013.

[4] Heyde, S. \& Lindner, J., Investigations on historic grey cast-iron columns. Proceedings of EUROSTEEL 2008, eds R. Ofner, D. Beg, J. Fink, R. Greiner \& H. Unterweger, ECCS: Southampton, Brussels, pp. 1545-1550, 2008.

[5] Rondal, J. \& Rasmussen, K.J.R., On the Strength of Cast Iron Columns, Brookhaven National Laboratory, The University of Sydney, Department of Civil Engineering, 24 pp., 2003.

[6] Rondal, J. \& Rasmussen, K.J.R., On the strength of cast iron columns. Journal of Constructional Steel Research, 60(9), pp. 1257-1270, 2004. DOI: 10.1016/ j.jcsr.2003.12.006.

[7] Heyde, S. \& Lindner, J., Historic grey cast-iron columns: Analysis of structural safety and upgrade. Stahlbau, 78(11), pp. 800-814, 2009. DOI: 10.1002/stab.200910096.

[8] Sykora, M., Holicky, M., Markova, J. \& Senberger, T., Probabilistic Reliability Assessment of Existing Structures (Focused on Industrial Heritage Buildings), Czech Technical University in Prague, CTU Publishing House: Prague, 108 pp., 2016.

[9] Kühn, B., Helmerich, R., Nussbaumer, A., Günther, H. \& Herion, S., Assessment of Existing Steel Structures: Recommendations for Estimation of Remaining Fatigue Life, JRC, 89 pp., 2007.

[10] Brych, I., Holicky, M., Jung, K. \& Sykora, M., Uncertainty in resistance models for historic cast-iron columns. Proceedings of Sustainable City X, eds C.A. Brebbia \& W.F. Florez-Escobar, WIT Press: Southampton and Boston, pp. 473-482, 2015.

[11] Brych, I. \& Sykora, M., Assessment of cast-iron columns using analytical models. Applied Mechanics and Materials, 821, pp. 782-788, 2016. DOI: 10.4028/ www.scientific.net/AMM.821.782.

[12] Caspeele, R., Sykora, M., Allaix, D.L. \& Steenbergen, R., The design value method and adjusted partial factor approach for existing structures. Structural Engineering International, 23(4), pp. 386-393, 2013.

DOI: $10.2749 / 101686613 X 13627347100194$.

[13] Holicky, M., Sykora, M. \& Retief, J.V., Assessment of model uncertainties for structural resistance. Probabilistic Engineering Mechanics, 45, pp. 188-197, 2016. DOI: 10.1016/j.probengmech.2015.09.008.

[14] Nadolski, V. \& Sykora, M., Uncertainty in resistance models for steel members. Transactions of the VSB - Technical University of Ostrava, Civil Engineering Series, 14(2), pp. 26-37, 2014. DOI: 10.2478/tvsb-2014-0028.

[15] Nadolski, V. \& Sykora, M., Model uncertainties in resistances of steel members. Proceedings of ESREL 2015, eds L. Podofillini, B. Sudret, B. Stojadinovic, E. Zio \& W. Kröger, Taylor and Francis/Balkema: Leiden, pp. 4189-4195, 2015.

[16] Holický, M., Tanner, P., Steenbergen, R., Nuti, C., Shave, J. \& Schnell, J., Assessment of Existing Structures (final draft of the technical specification, Apr. 2018), CEN TC250/WG2.T1, 40 pp., 2018.

[17] fib COM3 TG3.1, Partial Factor Methods for Existing Structures (fib bulletin 80, recommendation), fib, 129 pp., 2016.

[18] Sykora, M., Holicky, M., Jung, K. \& Diamantidis, D., Target reliability for existing structures considering economic and societal aspects. Structure and Infrastructure Engineering, 13(1), pp. 181-194, 2016. DOI: 10.1080/15732479.2016.1198394.

[19] Steenbergen, R.D.J.M., Sykora, M., Diamantidis, D., Holicky, M. \& Vrouwenvelder, A.C.W.M., Economic and human safety reliability levels for existing structures. Structural Concrete, 16, pp. 323-332, 2015. DOI: 10.1002/suco.201500022. 
82 High Performance and Optimum Design of Structures and Materials III

[20] Jung, K., Markova, J., Pokorny, P. \& Sykora, M., Material Properties of heritage wrought steel structure based on tests. International Journal of Heritage Architecture, 2(1), pp. 128-137, 2018. DOI: 10.2495/HA-V2-N1-128-137.

[21] Eberhardt, S. \& Pospíšil, M., Assessing cultural monuments: identifying and evaluating heritage values of masonry and cast-iron structures (under review). International Journal of Architectural Heritage, 2018. 